Conclusions False positive diagnosis of advanced fibrosis in NAFLD patients can be reduced and unnecessary liver biopsy can potentially be avoided by repeat LSM.

\section{IDDF2019-ABS-0273 HEPATIC RESECTION VERSUS TRANSCATHETER ARTERIAL CHEMOEMBOLIZATION IN RESECTABLE INFILTRATIVE HEPATOCELLULAR CARCINOMA: A PROPENSITY SCORE WEIGHTED LANDMARK STUDY}

Yuanqi Wang*, Xiao Han, Shuling Chen, Sui Peng, Zhenwei Peng, Ming Kuang, Jiaming Lai. The First Affiliated Hospital of Sun Yat-sen University, China

\subsection{6/gutjn|-2019-IDDFabstracts.301}

Background Patients with infiltrative hepatocellular carcinoma (iHCC) have a worse prognosis than those with other types of HCC and limited choices of treatments. The efficacy of hepatic resection in iHCC patients was unclear, especially comparing with other treatments. We aim to compare the efficacy of hepatic resection (HR) and transcatheter arterial chemoembolization (TACE) for patients with resectable iHCC

Methods We retrospectively enrolled patients with resectable iHCC who were treated by HR or TACE from four clinical centers. Their overall survival (OS) time was calculated and compared by Log-Rank test. A propensity score-matched (PSM) analysis was performed to reduce selection bias.

Results From January 2010 to December 2017, 178 patients with resectable iHCC were collected (124 patients received HR and 54 patients received TACE) and entered into 6,9,12-month landmark analysis. The median overall survival (OS) time was significantly longer in patients treated by HR than TACE (19 vs 11 months, $p=0.0041)$. Landmark analysis limiting survivors after 6, 9, 12 months also showed the benefit of HR over TACE in multi-variables COX regression (all $\mathrm{p}<0.01$ ). Patients with tumors located in both liver lobes or portal hypertension tended to receive HR rather than TACE. After propensity score matched, 46 pairs were compared and HR obtained better overall survival than TACE (median OS time: 29 vs 11 months, $\mathrm{p}=0.001$ ). The multi-variables analysis indicated vascular invasion as an independent predictor for worse OS in 6-month landmark subgroup(HR: 2.231, 95\%CI: 1.364-3.649, $\mathrm{p}=0.001$ ), especially for patients with the major trunk of portal vein involved.

Conclusions HR might be an optimal choice for patients with resectable iHCC due to its better survival benefit than TACE. Patients with trombosis in the major trunk of portal vein need multiple department team discussion to decide which therapy to be performed.

\section{IDDF2019-ABS-0280 PREDICTORS OF SURVIVAL FOR HEPATOCELLULAR CARCINOMA TREATED WITH ULTRASOUND-GUIDED PERCUTANEOUS RADIOFREQUENCY ABLATION}

Virgilio Jr Lo*, Engelbert Simon Perillo, Stepen Wong. University of Santo Tomas Hospital, Philippines

\subsection{6/gutjnl-2019-IDDFabstracts.302}

Background Radiofrequency ablation (RFA) is a widely used technique for treating hepatocellular carcinoma (HCC). Based on current data, tumor size and liver function are predictors of survival for HCC. Predictors of Survival in the Philippines may be different from other countries. We aimed to determine the independent predictors of survival for HCC patients undergoing RFA.

Methods RFA was performed on 181 consecutive HCC patients from June 2007 to November 2018. Survival was estimated using Kaplan-Meier and multivariate analysis of survival predictors were analyzed using Cox regression.

Results After a median follow-up of 19.9 months, the 1-,3- and 5 -year overall survival rates were $81.4 \%, 53.7 \%$ and $30.4 \%$, respectively. Local tumor progression and intrahepatic distant recurrence were observed in $42.5 \%$ (77/181) and 30.9\% (56/ 181), respectively. Patients with serum albumin $\geq 3.5 \mathrm{~g} / \mathrm{L}$ had better survival compared to serum albumin $<3.5 \mathrm{~g} / \mathrm{L} \quad(1,3 \& 5$ years: $=69.5 \%, 47.2 \%, 38.9 \%$ vs $39.1 \%, 0 \%, 0 \% ; p<0.001-($ figure 1). Other factors associated with survival on univariate analysis included: Cirrhosis $(\mathrm{p}=0.0001)$, Child-pugh class $(\mathrm{p}=$ $0.026)$, RFA time $(p=0.011)$, Platelet count $<150 \times 10^{9} / \mathrm{L} \quad(\mathrm{p}=$ $0.002)$, INR $(p<0.001)$ and total bilirubin $(p=0.026)$. The sole independent predictor of survival on multivariate analysis was serum albumin $(\mathrm{OR}=3.40 ; 95 \% \mathrm{CI}=0.196-0.442 ; \mathrm{p}<0.001)$.

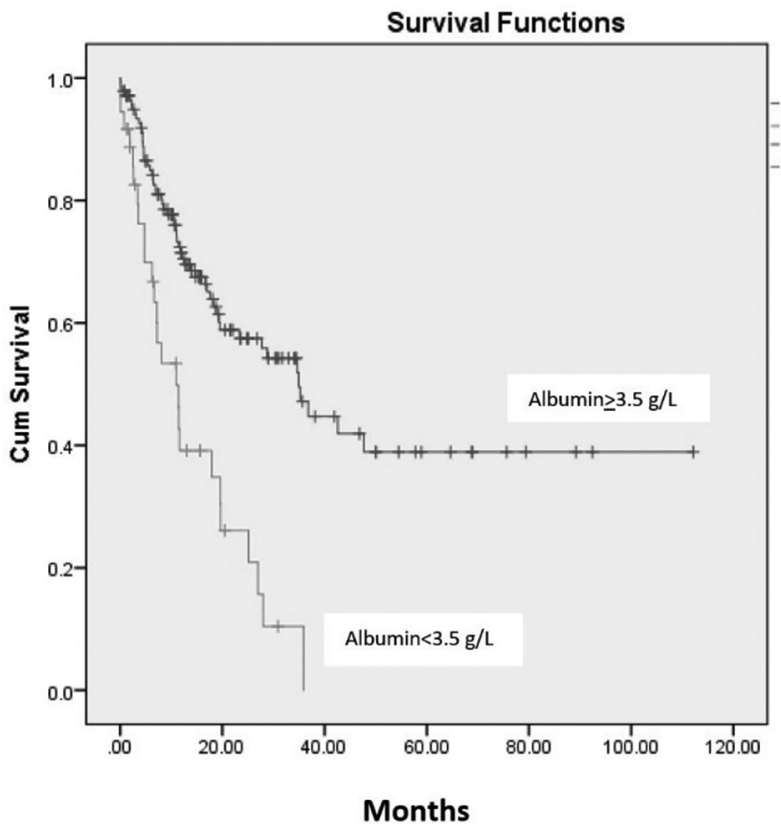

Abstract IDDF2019-ABS-0280 Figure 1

Conclusions In patients with HCC treated with RFA, serum albumin $\geq 3.5 \mathrm{~g} / \mathrm{L}$ appear to result in better survival.

\section{IDDF2019-ABS-0281 PREDICTORS OF TECHNIQUE EFFECTIVENESS FOR ULTRASOUND-GUIDED PERCUTANEOUS RADIOFREQUENCY ABLATION FOR HEPATOCELLULAR CARCINOMA}

Virgilio Jr Lo*, Virgilio Jr Lo, Engelbert Simon Perillo, Stephen Wong. University of Santo Tomas Hospital, Philippines

10.1136/gutjnl-2019-IDDFabstracts.303

Background Radiofrequency ablation (RFA) is a widely used technique for treating hepatocellular carcinoma (HCC). 